\title{
Intradural plasmacytoma originating from the filum terminale in the setting of multiple myeloma: illustrative case
}

\author{
Jamieson Glasser, BS, ${ }^{1}$ and Ryan Glasser, $\mathrm{MD}^{2}$ \\ ${ }^{1}$ Florida State University College of Medicine, Sarasota, Florida; and ${ }^{2}$ Clinical Sciences, Florida State University College of Medicine, Sarasota, Florida
}

\begin{abstract}
BACKGROUND Multiple myeloma (MM) has the propensity to spread to vertebral bodies; however, extramedullary plasmacytomas involving the central nervous system are extremely rare.

OBSERVATIONS The authors report the first intradural extramedullary plasmacytoma in the lumbar region of the spine in a patient with preexisting MM. They present a 50-year-old female with severe back and radicular pain and progressive neurological deficit.
\end{abstract}

LESSONS MM is typically treated with chemotherapy and radiation therapy. However, in this unique case, resection proved to be a key part of treatment.

https://thejns.org/doi/abs/10.3171/CASE20106

KEYWORDS intradural extramedullary; multiple myeloma; plasmacytoma; spinal tumor

Central nervous system (CNS) involvement in multiple myeloma (MM) is extremely rare. Most cases are from adjacent vertebral body disease extending into the epidural space or, less commonly, leptomeningeal disease. An intradural plasmacytoma that does not extend from an adjacent vertebral body has not been reported. We present a case of intradural plasmacytoma that appeared to arise from the filum terminale and caused severe compression of the cauda equina.

\section{Illustrative Case}

A 50-year-old female with a history of MM for 3 years presented with back pain and right-side sciatica for the past 2 weeks. She lives independently and maintains an active lifestyle. Since her diagnosis, she has been undergoing treatment at an academic center where she had a bone marrow transplant 3 years prior and a chimeric antigen receptor T-cell transplant 1 year prior. A fluorodeoxyglucose positron emission tomography (PET)/computed tomography (CT) scan 3 months prior did not show evidence of an intradural tumor or progressive disease. She has had lesions in the jaw, calvaria, and ribs, but no spinal involvement. The back and radicular pain worsened, and she noted numbness and weakness in both legs. She also developed progressive difficulty in ambulating and urinary retention. Physical examination was normal except for $4 / 5$ plantar flexion, $3 / 5$ dorsiflexion flexion, and decreased light touch sensation distal to the knees bilaterally.

Magnetic resonance imaging (MRI) with and without contrast was performed of the cervical, thoracic, and lumbar regions. These studies revealed diffuse abnormal bone marrow signal throughout the axial spine and adjacent tumors arising from multiple ribs, but no other intradural disease. Additionally, there was no abnormal leptomeningeal or dural enhancement identified on total axis MRI. A large intradural tumor was seen at L3 (Fig. 1). This intensely enhanced with contrast, but there was no adjacent bony disease or dural base. The tumor filled the spinal canal (Fig. 2) with the nerve roots draped around the periphery of the tumor (Fig. 3).

\section{Operation}

An L3 and partial L4 laminectomy was performed. After durotomy, a large grayish tumor that was highly vascular was encountered. There was no dural attachment of the mass. The tumor arose from a thick fibrous band with a large vessel on the surface, which is frequently seen on the posterior aspect of a thickened filum terminale (Fig. 4). This structure and all surrounding nerves were extensively studied with intraoperative nerve stimulation. There was no response to stimulation of the structure, which was thought to be consistent with

ABBREVIATIONS CNS = central nervous system; CSF = cerebrospinal fluid; $\mathrm{CT}=$ computed tomography; IDEM = intradural extramedullary; $\mathrm{MM}=$ multiple myeloma; $\mathrm{MRI}=$ magnetic resonance imaging; $\mathrm{PET}=$ positron emission tomography; $\mathrm{RT}$ = radiation therapy.

INCLUDE WHEN CITING Published February 8, 2021; DOI: 10.3171/CASE20106.

SUBMITTED November 11, 2020. ACCEPTED December 7, 2020.

(C) 2021 The authors, CC BY-NC-ND 4.0 (http://creativecommons.org/licenses/by-nc-nd/4.0/), 


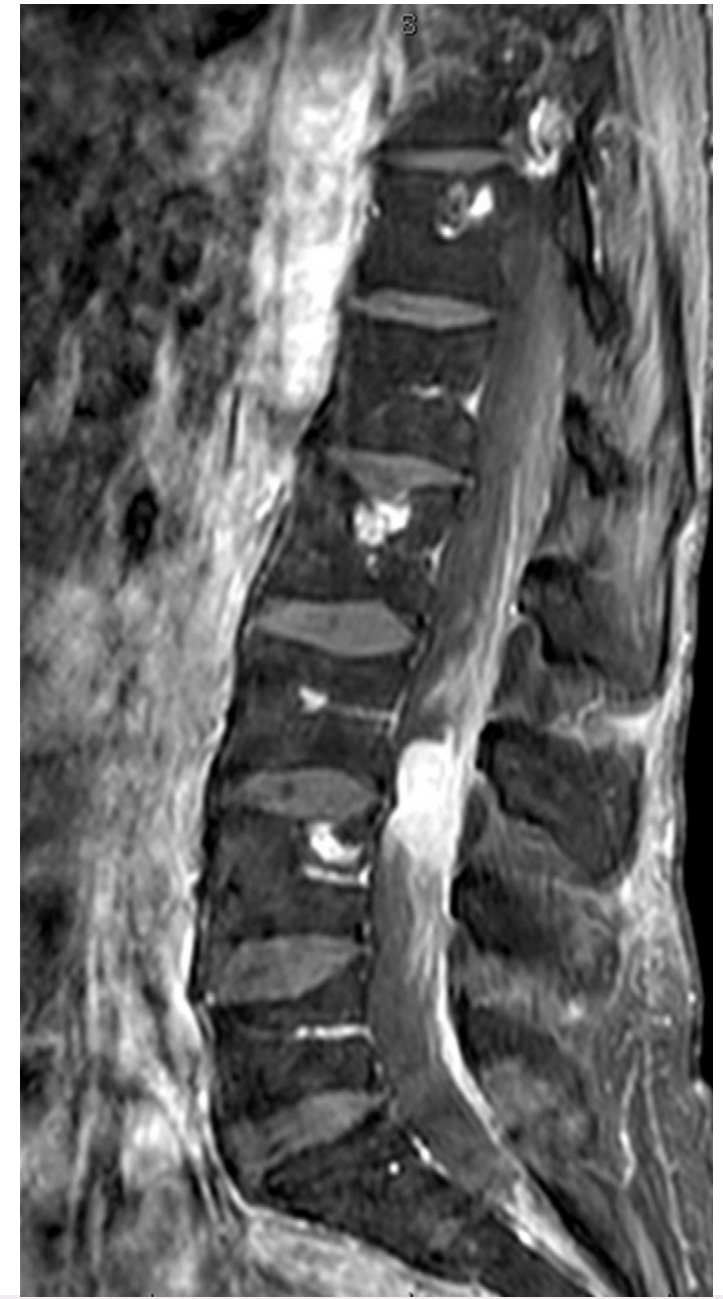

FIG. 1. Enhanced T1-weighted sagittal MRI of the lumbar spine reveals a large intradural tumor behind the body of $L 3$.

the filum. All nerves reliably responded to stimulation. This part of the tumor was completely resected. A portion of the tumor, however, invaded the right $L 4$ nerve root (Fig. 5), confirmed by intraoperative nerve monitoring. This was cut away from the nerve root. The entire tumor was removed, except for a small portion that was invading the right $L 4$ nerve.

\section{Pathological Findings}

There were numerous clonal plasma cells identified with a plasma cell count of $46 \%$, as well as an inverted CD4:CD8 ratio. These findings are consistent with the diagnosis of plasmacytoma.

\section{Postoperative Course}

The patient had immediate relief of her severe radicular pain. She also recovered from sensory loss, her strength returned to normal, and her urinary symptoms resolved. She went on to undergo adjuvant radiotherapy with the dosing of 20 Gy in 5 fractions. At a 6 -month followup, she had no evidence of intradural disease and continues to do well. She remains on maintenance chemotherapy.

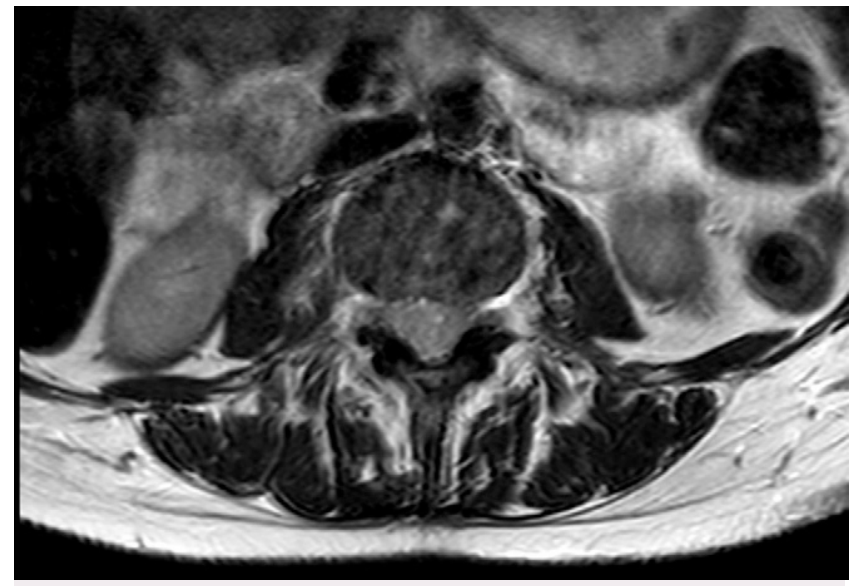

FIG. 2. T2-weighted axial MRI at the level of the mass shows tumor nearly filling the entire spinal canal.

\section{Discussion \\ Observations}

Spinal tumors are primarily defined by region and location within the spine. Intradural extramedullary (IDEM) tumors account for $30 \%$ of spinal tumors. Meningiomas, schwannomas, neurofibromas, and ependymomas are the most common in this location. ${ }^{1}$ Plasmacytomas within the epidural space can cause compression of the spinal cord and neurological symptoms. Between $5 \%$ and $10 \%$ of patients with MM develop neurological symptoms; however, a primary growth from a vertebral body is usually responsible. ${ }^{2-4}$

Intradural plasmacytomas are extremely rare and almost unheard of in the lumbar region, whereas $80 \%$ of plasmacytomas found outside extramedullary bone occur in the head and neck and the upper respiratory tract. ${ }^{5}$ Plasmacytomas that involve the CNS are more often intracranial and dural based. ${ }^{3}$ In a review of the literature, there were 6 other intradural plasmacytomas originating in the spine.,2, 2,7

MM history and status is an important consideration when working up IDEM tumors. CNS involvement occurs in only about $1 \%$ of patients with MM, and plasma cell accumulation in the cerebrospinal fluid (CSF) can be present in advanced MM. ${ }^{8,9}$ However, even without a previous diagnosis of MM in a case of IDEM tumor, a solitary plasmacytoma could be the presenting sign of systemic disease. Only $1 \%$ to $2 \%$ of patients with MM have disease outside the bone when diagnosed, and $8 \%$ develop it later as the disease progresses. ${ }^{10}$ The usual presenting age for a plasmacytoma is 55 years, and an earlier age of presentation is associated with MM. ${ }^{6}$ Additionally, previously reported plasma cell involvement of the dura has a predilection in women with a mean age of 57.5 years. ${ }^{9}$

Singh et al. ${ }^{6}$ report an IDEM tumor in the lumbar region at L2. This presented with radiculopathy and was treated similarly to our case with laminectomy, excision, and postoperative radiation therapy (RT). In contrast, theirs was a solitary plasmacytoma that arose from the ventral dura and showed adjacent vertebral body hyperostosis. ${ }^{6}$ Hyperostotic reaction and/or tumor calcification have been reported as signs of plasmacytoma. ${ }^{11}$ There is no consensus on how plasma cells spread to the dura mater, but the case reported by Singh et al. ${ }^{6}$ would support the hypothesis that plasma cells spread to the dura mater through contiguous bone lesions. However, our case did not show contiguous bone 


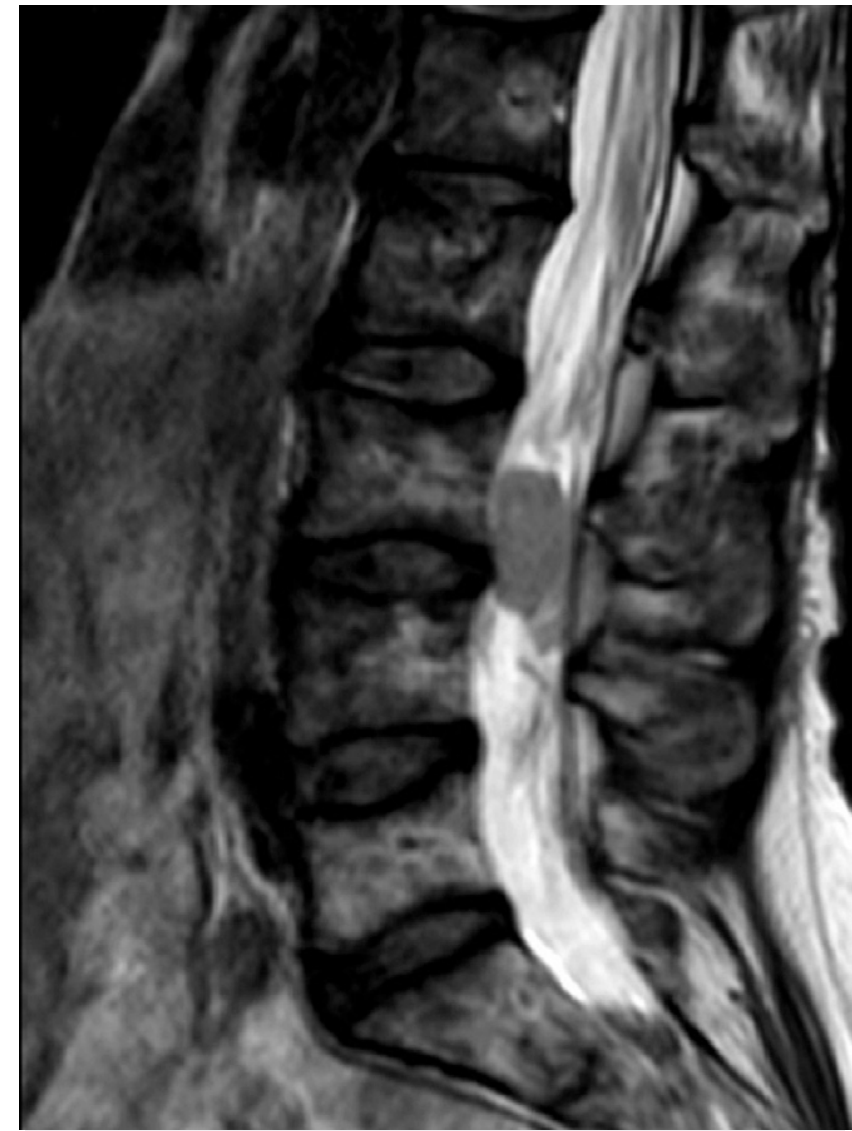

FIG. 3. T2-weighted sagittal MRI of the lumbar spine shows the relationship of the tumor to the cauda equina.

lesions and so would be better explained by the hypothesis that tumor cells spread to the dura through microscopic dural vessels, which also has been supported in the presentation of cranial plasmacytomas and reported by Haegelen et al. ${ }^{9}$

Clinical presentations are variable and can range from pain with paraparesis to only radiculopathy. ${ }^{2,6}$ The patient in this case presented with similar findings but additionally had urinary retention.

Radiological images for plasmacytomas tend to be nonspecific and can be mistaken for other tumors such as neurofibromas. ${ }^{6}$ It is also important to discuss the role of PET/CT scans in the diagnostic workup of suspected plasmacytomas. A routine PETscan is obtained for monitoring MM disease status. However, it has limitations and may not reveal intradural disease, as in this case with the patient showing no signs of intradural disease on a scan 3 months prior to presentation. If CNS involvement is suspected in a patient with MM, a PET/CT is recommended along with complete neuroimaging and possible testing of the CSF to look for monoclonal plasma cells. ${ }^{8}$ This has been exemplified in a previously reported case of relapsing MM. After hematological remission with interferon and RT, a patient relapsed with neurological symptoms and showed intradural disease via plasma cells and lgG lambda-type M protein being detected in the CSF.,12 That is a related presentation yet vastly different from the current case because there was no discrete plasmacytoma, rather just plasma cells and evidence of MM in the CSF. Furthermore, the gamut of tests ordered in the workup of patients depends on patient presentation and clinical context.

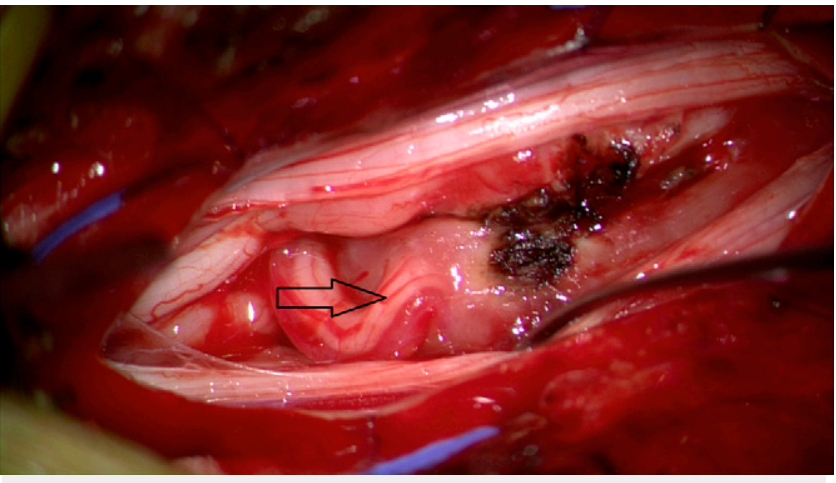

FIG. 4. Intraoperative photograph demonstrates tumor arising from filum terminale (arrow).

Intraoperative and gross appearance of the tumors, as first detailed by Sod and Wiener in 1959, can resemble typical meningiomas and neurofibromas and can also involve nerve roots. ${ }^{2,6}$ They report the tumor arising from the ventral dura and invading adjacent nerve roots. In this current case, the tumor invaded the $L 4$ nerve root but appeared to arise from the filum terminale and had to carefully be excised with some tumor being left behind to preserve nerve function.

Following resection, regardless of whether the tumor was totally or partially excised, adjuvant local RT was administered in 3 of the 6 known cases and was not reported in the other 2.2,6 Because plasmacytomas are radiosensitive, adjuvant radiotherapy can increase the local cure rate after surgery. ${ }^{11,13}$ Because of the rarity of intradural plasmacytomas and the inexperience of clinicians in treating it, there is no consensus on what is the optimal RT dose, but a general rule is 40-50 Gy for macroscopic disease and 36-40 Gy for microscopic disease. In some cases of complete resection, observing and following the patient may be all that are warranted. ${ }^{13}$ In this case, the patient received $20 \mathrm{~Gy}$ in 5 fractions, slightly less than the recommended dose, but this was thought to be effective because of the near-total resection.

Surgery absolutely plays a role in the management of intradural plasmacytomas when severe neurological symptoms exist. Debulking or removal of the tumor can play a vital role in decompressing nerve roots. ${ }^{7}$ This was exemplified in this case because it led to the immediate

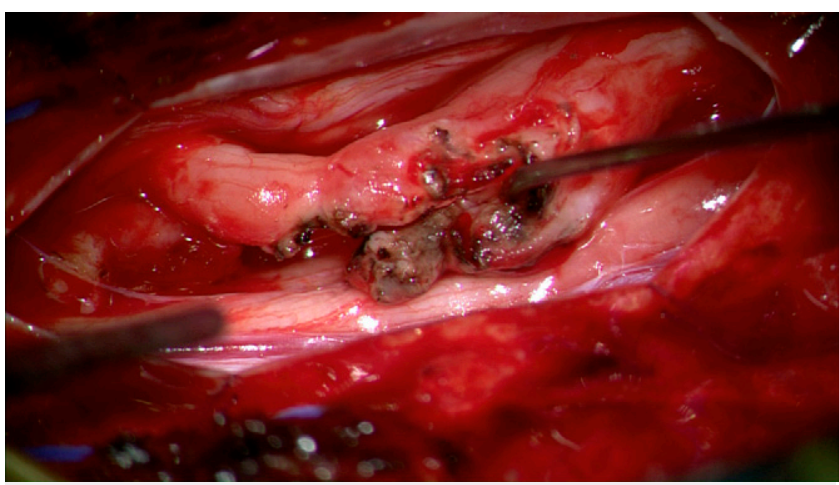

FIG. 5. Intraoperative photograph shows tumor infiltrating the right L4 nerve root. 
resolution of symptoms and return to a good quality of life for this patient. This is even more paramount considering the grim prognosis that extramedullary presentation of MM brings, with median survival rates of 2-8 months. ${ }^{7}$ More specifically, of the reported cases in the literature of spinal involvement of $\mathrm{MM}$, the survival has ranged from 1 to 11 months but is limited by extremely small sample sizes. ${ }^{7}$

\section{Lessons}

Advanced stages of MM can impact the CNS and cause severe neurological symptoms. The MRI appearance of intradural plasmacytoma may have a similar appearance to other more common IDEM tumors in the lumbar spine. Chemotherapy and RT play significant roles in the treatment of MM. These treatment modalities may not afford as immediate symptom resolution as surgery did in this case. When severe neurological symptoms are present, surgical treatment is highly effective in alleviating pain and neurological deficits.

\section{References}

1. Ottenhausen M, Ntoulias G, Bodhinayake I, et al. Intradural spinal tumors in adults-update on management and outcome. Neurosurg Rev. 2019;42(2):371-388.

2. Sod LM, Wiener LM. Intradural extramedullary plasmacytoma; case report. J Neurosurg. 1959;16(1):107-109.

3. Zazpe I, Caballero C, Cabada T, et al. Solitary thoracic intradural extramedullary plasmacytoma. Acta Neurochir (Wien). 2007; 149(5):529-532.

4. Hans FJ, Geibprassert S, Krings T, et al. Solitary plasmacytoma presenting as an intramedullary mass of the cervical cord. J Neurol Surg A Cent Eur Neurosurg. 2013;74(suppl 1):e13-e17.

5. Gao B, Wang X. An extramedullary plasmacytoma originating from the thoracic spinal cord: magnetic resonance imaging findings. Case report. J Neurosurg Spine. 2007;6(1):57-59.

6. Singh S, Kumar A, Bhaisora KS, et al. An unusual case of solitary spinal intradural plasmacytoma: The unforeseen challenge. J Craniovertebr Junction Spine. 2019;10(3):192-196.
7. Di L, Huang K, Kesayan T, et al. Multiple myeloma presenting as an intramedullary spinal cord tumor: a case report and review of the literature. J Med Case Reports. 2020;14(1):189.

8. Sevcikova S, Minarik J, Stork M, et al. Extramedullary disease in multiple myeloma - controversies and future directions. Blood Rev. 2019;36:32-39.

9. Haegelen C, Riffaud L, Bernard M, et al. Dural plasmacytoma revealing multiple myeloma. Case report. J Neurosurg. 2006; 104(4):608-610.

10. Rajkumar SV. Multiple myeloma: 2018 update on diagnosis, riskstratification, and management. Am J Hematol. 2018;93(8): $981-1114$.

11. Benli K, Inci S. Solitary dural plasmacytoma: case report. Neurosurgery. 1995;36(6):1206-1209.

12. Hayakawa $\mathrm{H}$, Obama K, Tara M. Intradural recurrence of multiple myeloma during the hematological complete remission. Article in Japanese. Rinsho Ketsueki. 2002;43(11):1009-1013.

13. Strojan $P$, Soba E, Lamovec J, et al. Extramedullary plasmacytoma: clinical and histopathologic study. Int J Radiat Oncol Biol Phys. 2002;53(3):692-701.

\section{Disclosures}

The authors report no conflict of interest concerning the materials or methods used in this study or the findings specified in this paper.

\section{Author Contributions}

Conception and design: both authors. Acquisition of data: both authors. Analysis and interpretation of data: both authors. Drafting the article: both authors. Critically revising the article: both authors. Reviewed submitted version of manuscript: both authors. Approved the final version of the manuscript on behalf of both authors: J Glasser.

\section{Correspondence}

Jamieson Glasser: Florida State University College of Medicine, Sarasota, FL. jsg14@med.fsu.edu. 